\title{
T lymphoblastic leukemia/lymphoma and human immunodeficiency virus infection
}

\author{
Kamraan Z. Gill • Shafinaz Hussein • Yuxia Jia • \\ Vundavalli V. Murty • Govind Bhagat • Bachir Alobeid
}

Received: 27 June 2011 / Accepted: 4 July 2011 /Published online: 21 July 2011

(C) Springer-Verlag 2011

\section{Dear Sir,}

Human immunodeficiency virus-infected (HIV+) individuals are at high risk for developing certain hematologic malignancies such as diffuse large B-cell lymphoma and Burkitt lymphoma. Advances in therapeutic management have improved the long-term survival of HIV-infected individuals leading to increases in the incidence of other neoplasms that were hitherto considered rare in this patient population. Nevertheless, T lymphoblastic leukemia/lymphoma (T-ALL/ LBL) remains a rare occurrence in patients with HIV infection; only five cases have been previously reported [1-5]. Here, we describe an additional case of T-ALL/LBL occurring in the setting of HIV infection.

A 55-year-old man with a history of HIV/AIDS presented with weakness, fever, weight loss, and a neck mass for 1 month. He was diagnosed with HIV/AIDS 20 years prior to presentation, and his course had been complicated by numerous opportunistic infections. He had been treated with HAART since the time of original diagnosis, but he was reported to be noncompliant. His

\footnotetext{
K. Z. Gill · S. Hussein • V. V. Murty • G. Bhagat • B. Alobeid $(\bowtie)$ Departments of Pathology and Cell Biology, Columbia University Medical Center and New York Presbyterian Hospital, 630 W. 168 St., VC14-229,

New York, NY 10032, USA

e-mail: ba2024@columbia.edu

K. Z. Gill

e-mail: kzg2101@columbia.edu

Y. Jia

Division of Hematology/Oncology, Columbia University Medical Center and New York Presbyterian Hospital,

New York, NY, USA
}

most recent CD4 count and viral load were $218 / \mu \mathrm{L}$ (normal range $393-1489 / \mu \mathrm{L}$ ) and 68 copies $/ \mathrm{mL}$ (by PCR assay, COBAS AmpliPrep/COBAS TaqMan HIV-1 Test Kit, Version 2.0), respectively. His physical exam was significant for fever $\left(39.5^{\circ} \mathrm{C}\right)$, lymphadenopathy (submental, cervical, and inguinal), and skin rash consisting of erythematous papules and hyperpigmented macules on the trunk and proximal extremities. Laboratory studies showed a hemoglobin concentration of $5.1 \mathrm{~g} / \mathrm{dL}$ (normal range $13.3-16.2 \mathrm{~g} / \mathrm{dL})$, a white blood cell count of $4.0\left(\times 10^{9} / \mathrm{L}\right.$, normal range 3.54-9.06), and a platelet count of $52,000 / \mu \mathrm{L}$ $\left(52 \times 10^{9} / \mathrm{L}\right.$, normal range $\left.165-415 \times 10^{9} / \mathrm{L}\right)$. CT scan revealed lymphadenopathy involving the neck, mediastinal, mesenteric, retroperitoneal, and inguinal lymph nodes.

A bone marrow biopsy revealed a markedly hypercellular marrow for age $(90 \%)$, the entire marrow space being replaced by an extensive infiltrate of blasts (Fig. 1a). The marrow aspirate also showed an extensive population of blasts, which accounted for $86 \%$ of all marrow nucleated elements. Flow cytometric analysis of the aspirate revealed a population of blasts that expressed CD34, TdT, cytoplasmic CD3, CD5, CD7, CD33, CD43, and HLA-DR and did not express surface CD3, CD2, CD4, CD8, CD1a, CD117, CD13, CD11c, surface TCR, or CD64 (Fig. 1b-d). This immunophenotype was diagnostic of T-ALL. A biopsy of a lymph node from the left neck showed extensive paracortical infiltrates of lymphoblasts, and the immunophenotype of these blasts was similar to that of the blasts in the bone marrow. The lymphoblasts were negative for EBV by EBVencoded RNA in situ hybridization. G band karyotype analysis of the bone marrow and lymph node samples revealed the translocation $\mathrm{t}(7 ; 14)(\mathrm{p} 15 ; \mathrm{q} 32)$, which, although rare, has been previously described in association with $\mathrm{T}$ - 
Fig. 1 Bone marrow biopsy and flow cytometry. a The bone marrow biopsy shows a hypercellular marrow with an extensive infiltrate of blasts (H\&E stain, $\times 200$ ). b-d Flow cytometric analysis of the bone marrow aspirate demonstrates expression of cytoplasmic CD3, TdT, CD7, partial CD5, and aberrant CD33, consistent with a T-cell lineage acute lymphoblastic leukemia

\section{a}

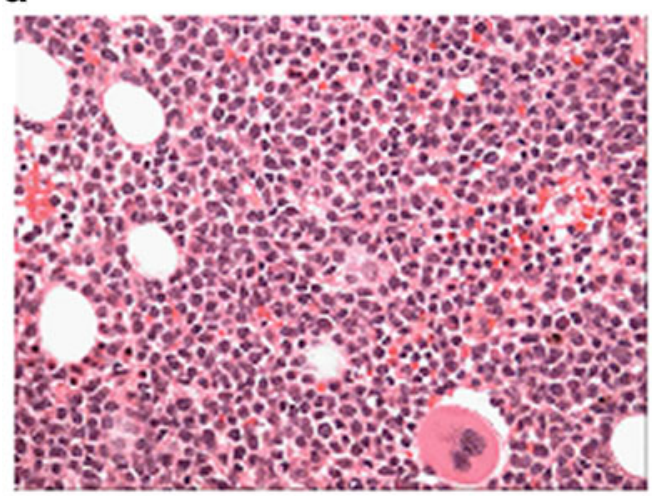

c

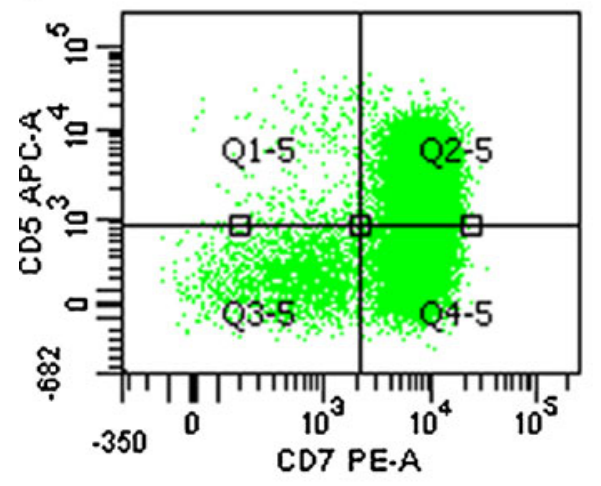

b

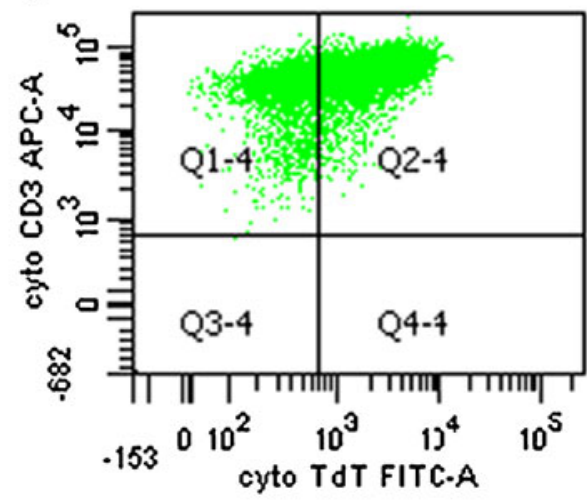

d

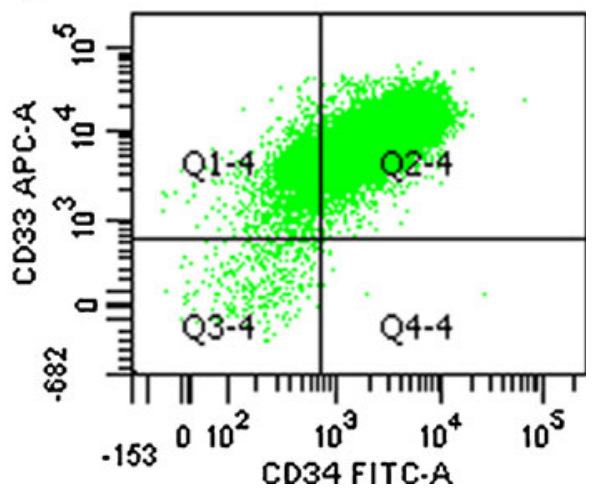

ALL/LBL [6]. A biopsy from one of the hyperpigmented skin lesions revealed leukemia cutis.

After diagnosis of T-ALL, the patient was started on a regimen of hyper-CVAD (hyperfractionated; cyclophosphamide, vincristine, doxorubicin (Adriamycin), and dexamethasone) with CNS prophylaxis. He completed one cycle of treatment but subsequently developed sepsis with vancomycin-resistant Enterococcus faecium and fungemia with Candida albicans and died 1 month after diagnosis of T-ALL. A repeat bone marrow biopsy was not performed.

Five cases of T-ALL/LBL occurring in the setting of HIV/AIDS have been previously reported [1-5]. Comparing these few cases to our own, there do not appear to be any distinguishing features of T-ALL/LBL occurring in the setting of HIV infection, except short survival. The survival ranged from 1 to 30 months with a mean of 10 months, which is shorter than the survival for T-ALL/ LBL occurring in the general population [7, 8]. This apparently decreased survival is likely related to the underlying immunocompromised state and comorbidities associated with HIV infection. Given the lack of distinguishing features and rarity, the concurrence of T-ALL/ LBL and HIV/AIDS is most likely coincidental, without any underlying pathogenetic relationship. As more cases accumulate in the literature, it would be interesting to assess in greater depth any differences between T-ALL/ LBL occurring in HIV/AIDS patients and cases occurring in the general population.

Conflicts of interest The authors declare that they have no conflict of interest.

\section{References}

1. Lorenzon D, Perin T, Bulian P et al (2009) Human immunodeficiency virus-associated precursor T-lymphoblastic leukemia/lymphoblastic lymphoma: report of a case and review of the literature. Hum Pathol 40:1045-1049

2. Lum GH, Cosgriff TM, Byrne R et al (1993) Primary T-cell lymphoma of muscle in a patient infected with human immunodeficiency virus. Am J Med 95:545-546

3. Geriniere L, Bastion Y, Dumontet C et al (1994) Heterogeneity of acute lymphoblastic leukemia in HIV-seropositive patients. Ann Oncol 5:437-440

4. Presant CA, Gala K, Wiseman C et al (1987) Human immunodeficiency virus-associated T-cell lymphoblastic lymphoma in AIDS. Cancer 60:1459-1461

5. Rujirojindakul P, Kayasut K, Rohitoprakarn M et al (2007) A unique case of transient spontaneous regression complicated with tumor lysis syndrome of T-cell lymphoblastic lymphoma in HIVinfected patient without antiretroviral therapy. J Med Assoc Thai 90:1930-1933 
6. Heerema NA, Sather HN, Sensel MG et al (1998) Frequency and clinical significance of cytogenetic abnormalities in pediatric T-lineage acute lymphoblastic leukemia: a report from the Children's Cancer Group. J Clin Oncol 16(4):1270-1278

7. Goldberg JM, Silverman LB, Levy DE et al (2003) Childhood Tcell acute lymphoblastic leukemia: the Dana-Farber Cancer
Institute acute lymphoblastic leukemia consortium experience. J Clin Oncol 21(19):3616-3622

8. Silverman LB, Stevenson KE, O'Brien JE et al (2010) Long-term results of Dana-Farber Cancer Institute ALL Consortium protocols for children with newly diagnosed acute lymphoblastic leukemia (1985-2000). Leukemia 24(2):320-334 